\title{
Hemorrhagic Infarction Originating From Vertebral Artery Stenosis Caused by an Osteophyte at the C5 Superior Articular Process
}

\author{
-Case Report- \\ Satoru TAKEUCHI, Tsutomu KAWAGUCHI, \\ Mitsuru NAKATANI, and Toyohiko ISU* \\ Department of Neurosurgery, Hakodate Red Cross Hospital, Hakodate, Hokkaido; \\ * Department of Neurosurgery, Kushiro Rosai Hospital, Kushiro, Hokkaido
}

\begin{abstract}
A 62-year-old man presented with right upper homonymous quadrihemianopsia. Magnetic resonance imaging showed hemorrhagic infarctions in the bilateral occipital lobes and infarction in the left cerebellar hemisphere. Digital subtraction angiography and three-dimensional computed tomography angiography revealed right vertebral artery (VA) stenosis caused by an osteophyte at the C5 superior articular process. The VA stenosis was aggravated by neck extension. The brain infarctions were attributed to embolism from the right VA stenosis. Unroofing of the anterior root of the right C4 transverse foramen, removal of the osteophyte at the superior articular process of $\mathrm{C5}$, and anterior fusion at $\mathrm{C4-5}$ were performed. His postoperative course was uneventful.
\end{abstract}

Key words: vertebral artery stenosis, osteophyte, hemorrhagic infarction

\section{Introduction}

Vertebrobasilar insufficiency may be caused by many types of lesion, but intrinsic lesions, such as atherosclerosis, are usually responsible. Extrinsic lesions are less common, ${ }^{1,5,6)}$ and often involve osteophytes at the uncocervical point from C6 to C2 causing compression of the vertebral artery (VA), ${ }^{1,3,4)}$ which tends to be exacerbated by neck rotation. We treated a patient with hemorrhagic infarction originating from right VA stenosis caused by an osteophyte at the C5 superior articular process.

\section{Case Report}

A 62-year-old man presented with a 2-week history of visual field defects. Physical examination detected right upper homonymous quadrihemianopsia. Head turning elicited no symptoms. He had no history of neck injury or excess neck movement.

Magnetic resonance (MR) imaging revealed hemorrhagic infarctions in the bilateral occipital lobes and infarction in the left cerebellar hemisphere (Fig. 1A, B). MR angiography showed stenosis of the right VA and hypoplasia of the left VA (Fig. 1C). Three-dimensional computed tomography (3D-CT) angiography disclosed right VA stenosis with back-to-front compression by an osteophyte at the C5 superior articular process (Fig. 2 ). Right vertebral angiography showed moderate stenosis at the C4-5 level (Fig. 3). The VA stenosis was exacerbated by neck extension but not by head rotation. Aortography failed to visualize the left VA due to hypoplasia (Fig. 1D). The diagnosis was brain infarction, probably due to embolism from the right VA stenosis caused by an osteophyte at the C5 superior articular process.

He wore a soft cervical collar to limit head turning and received antiplatelet drugs for 6 weeks preceding surgery. A right-sided anterior approach to the cervical spine was performed. The right VA was identified at the C3-4 and C4-5 levels with a Doppler

Received December 21, 2006; Accepted October 15, 2008

Author's present address: S. Takeuchi, M.D., Department of Neurosurgery, National Hospital Organization Disaster Medical Center, Tachikawa, Tokyo, Japan. 

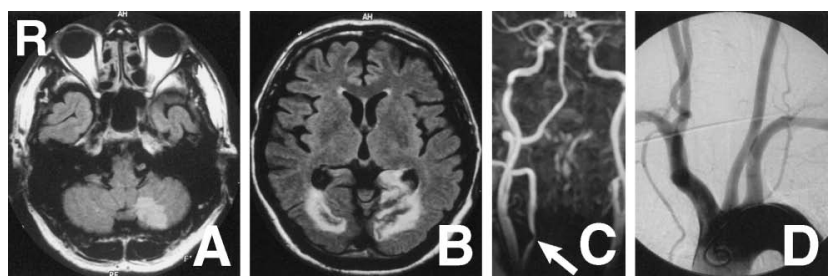

Fig. 1 A, B: Fluid-attenuated inversion recovery magnetic resonance images revealing hyperintense lesions in the bilateral occipital lobes and the left cerebellar hemisphere. C: Magnetic resonance angiogram showing stenosis of the right vertebral artery (arrow) and hypoplasia of the left vertebral artery. D: Aortogram not visualizing the left vertebral artery due to hypoplasia.
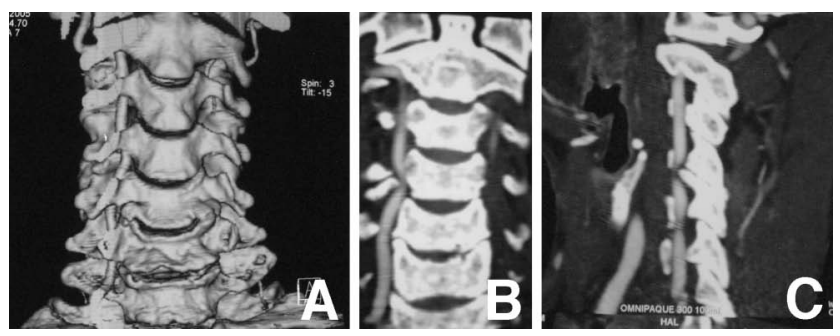

Fig. 2 Three-dimensional (A), coronal (B), and sagittal (C) computed tomography angiograms demonstrating severe stenosis and compression of the right vertebral artery from back to front by an osteophyte at the C5 superior articular process.
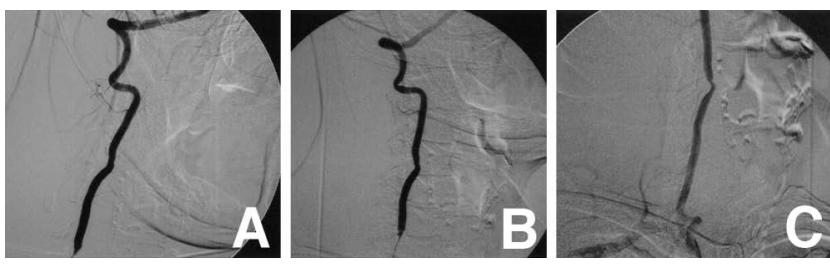

Fig. 3 Right vertebral angiograms showing moderate stenosis during flexion of the neck (A) and in the neutral position (B), and marked stenosis during neck extension (C).

probe. The anterior root of the $\mathrm{C} 4$ transverse foramen was unroofed using a high-speed drill and an ultrasonic surgical aspiration/irrigation system (SONOPET OST-2001; Miwatec Co., Ltd., Kawasaki, Kanagawa). Intraoperative angiography found no evidence of improvement in the stenosis. We used a SONOPET to resect the osteophyte at the C5 superior articular process that compressed the VA,
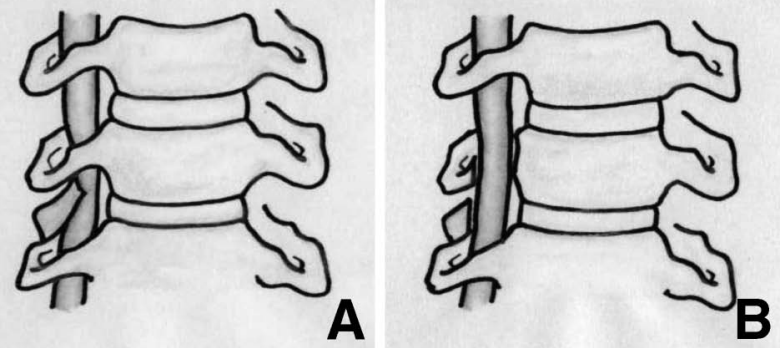

Fig. 4 A: Scheme illustrating stenosis of the right vertebral artery due to posterolateral-anteromedial compression by an osteophyte at the C5 superior articular process. B: Scheme illustrating the postoperative disappearance of stenosis of the right vertebral artery.
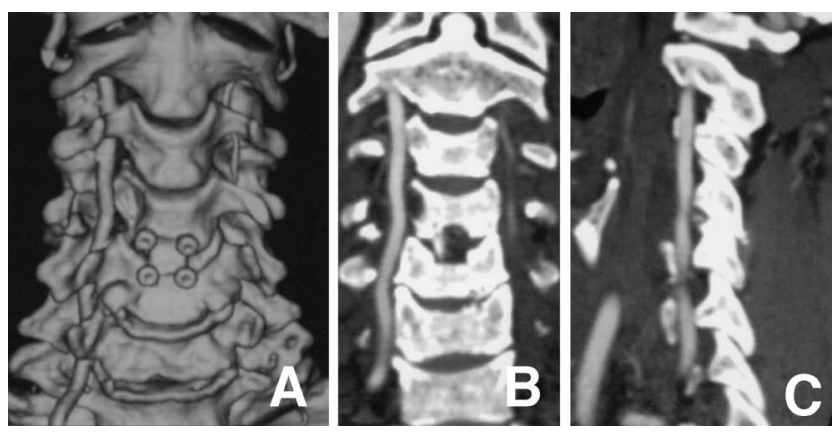

Fig. 5 Three-dimensional (A), coronal (B), and sagittal (C) computed tomography angiograms demonstrating complete disappearance of the severe vertebral artery stenosis and compression.

resulting in disappearance of the VA stenosis (Fig. 4). The VA stenosis was exacerbated by neck extension, so we performed anterior fusion at the C4-5 level.

No intraoperative complications occurred and his postoperative course was uneventful. He was discharged 20 days after surgery and was able to walk. 3D-CT angiography performed 1 month after operation showed no evidence of stenosis or compression of the right VA (Fig. 5). He remained free of complaints and the right VA remained patent at 3-year follow-up examination.

\section{Discussion}

Only one previous patient has presented with embolic infarction from VA stenosis attributable to cervical spondylosis, caused by an osteophyte located at 
the uncocervical point. ${ }^{7)}$ Stenosis and compression of the VA caused by an osteophyte at the superior articular process is extremely rare. Our patient presented with multiple brain infarcts with hemorrhagic characteristics, so we suspected an embolic etiology. MR and 3D-CT angiography were useful in reaching the correct diagnosis. 3D-CT angiography demonstrated the relationship between bone and vascular structures particularly well. We recommend that dynamic positional angiography be performed to assess the operative indications and methods. In our patient, the VA stenosis and compression were exacerbated by neck extension rather than head rotation because the osteophyte compressed the vessel from the posterior to the anterior.

Our surgical strategy involved unroofing of the anterior root of the $\mathrm{C} 4$ transverse foramen, resection of the osteophyte at the C5 superior articular process, and anterior fusion at the C4-5 level. We found that the SONOPET was useful for removing the anterior root and osteophyte adjacent to the $\mathrm{VA},{ }^{2)}$ and that intraoperative angiography could confirm the disappearance of the VA stenosis.

\section{References}

1) Bakay L, Leslie EV: Surgical treatment of vertebral artery insufficiency caused by cervical spondylosis. J
Neurosurg 23: 596-602, 1965

2) Kim K, Isu T: Surgical pitfalls of an ultrasonic bone curette(SONOPET) in spinal surgery. Neurosurgery 59(4 Suppl 2): ONS 390-393, 2006

3) Kuether TA, Nesbit GM, Clark WM, Barnwell SL: Rotational vertebral artery occlusion: A mechanism of vertebrobasilar insufficiency. Neurosurgery 41: 427-432, 1997

4) Nagashima C: Surgical treatment of vertebral artery insufficiency caused by cervical spondylosis. J Neurosurg 32: 512-521, 1970

5) Powers SR Jr, Drislane TM, Nevins S: Intermittent vertebral artery compression; a new syndrome. Surgery 49: 257-264, 1961

6) Sorensen BF: Bow hunter's stroke. Neurosurgery 2: 259-261, 1978

7) Yamaguchi S, Sakata K, Nakayama K, Shigemori M: [A case of embolic infarction originating from extracranial vertebral artery stenosis by cervical spondylosis at C5/6: Its pathogenesis and surgical treatment]. No Shinkei Geka 31: 1111-1116, 2003 (Jpn, with Eng abstract)

Address reprint requests to: Satoru Takeuchi, M.D., Department of Neurosurgery, National Hospital Organization Disaster Medical Center, 3256 Midori-cho, Tachikawa, Tokyo 190-0014, Japan. e-mail: satoru.t@tdmc.hosp.go.jp 